WHAT IS YOUR CALL?

\title{
Chronic vulvocrural dermatitis with burning and itching
}

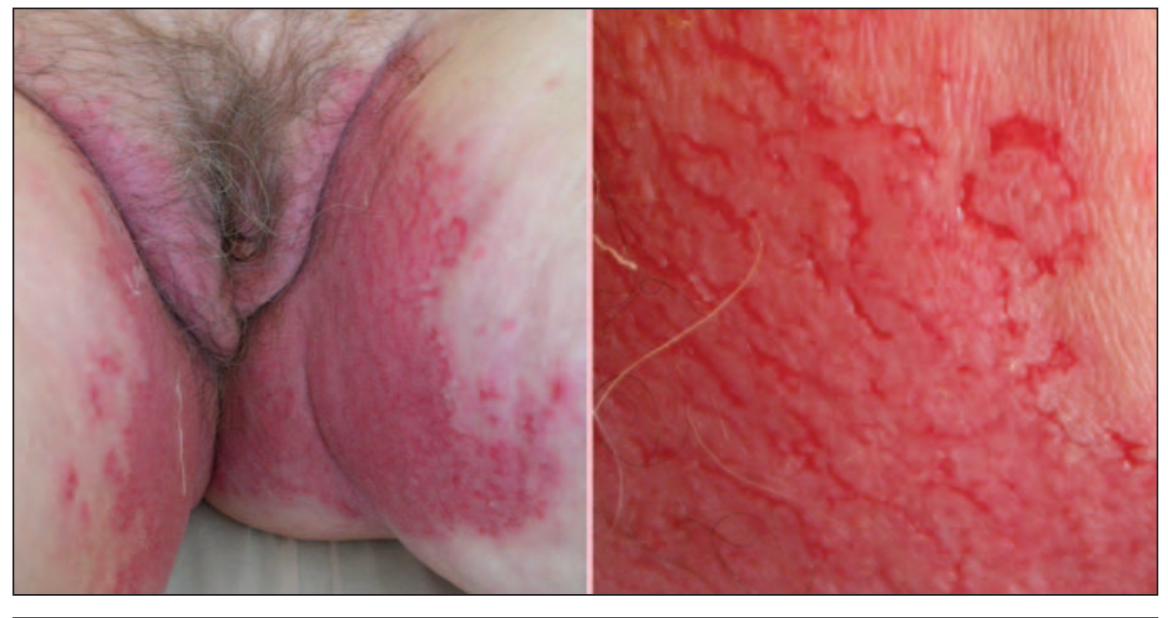

Figure 1: A widespread erythematous blistering eruption involving the whole vulva (left); well-demarcated fissured malodorous plaques in the inguinal region (right).

\section{$\mathrm{A}$} 73-year-old woman presented with an 18-year history of an intermittent recalcitrant groin rash, vulvar burning and dyspareunia. The rash started on her right labia majora and had spread gradually over the entire vulva, groin and perineum over a 2-week period (Figure 1). In addition, she had well-demarcated fissured malodorous plaques in her inguinal region. The patient's symptoms recurred seasonally, becoming more irritating during warm weather. Her symptoms had been treated with topical antifungals with short-term remission. In addition to the skin rash, our patient had white, longitudinal fingernail bands, and she reported that her son and daughter had similar eruptions localized to their forearms and axillae (Figure 2).

\section{What is your diagnosis?}

a. Intertrigenous candidiasis

b. Eczema

c. Recurrent herpes simplex

d. Inverse psoriasis

e. Hailey-Hailey disease

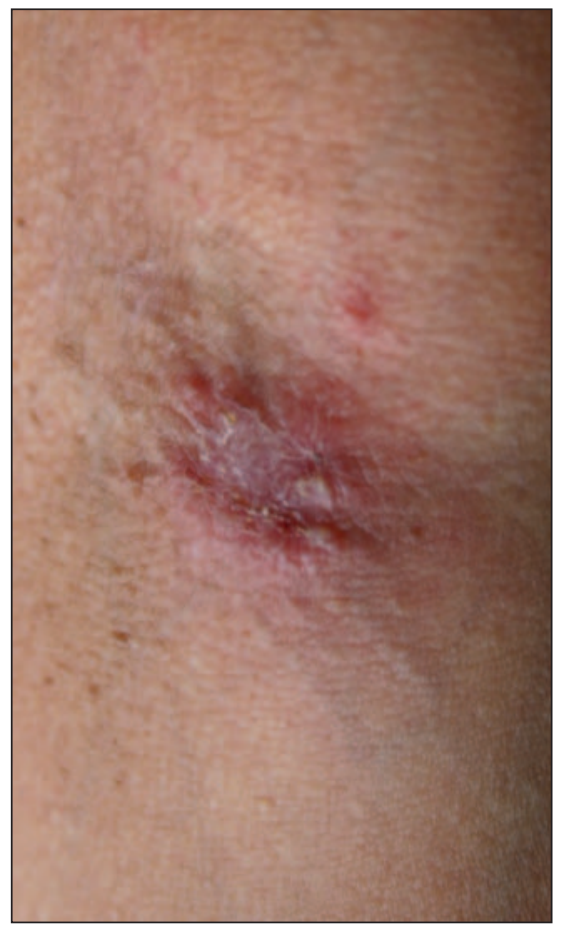

Figure 2: The patient's daughter had a crusted skin erosion with a peripheral scaling border on the flexural area of her forearm.

CMAJ invites submissions to "What is your call?" Clinical details (including images) are presented on the first page along with a multiple-choice question about the diagnosis. The answer and a brief discussion of the condition follow on the second page. We specifically invite submissions illustrating common or important radiographic and electrocardiographic diagnoses of appeal to a general audience. We allow up to 5 references and require authors to obtain consent from the patient for publication of his or her story (form available at www.cmaj.ca/authors/checklist.shtml). Submit manuscripts online at http://mc.manuscriptcentral.com/cmaj. 


\section{WHAT IS YOUR CALL?}

\section{Discussion}

The answer is (e) Hailey-Hailey disease or familial benign pemphigus. We made this diagnosis based on a positive family history (the patient's son and daughter had similar skin changes), nail changes (white streaks) and histopathological signs (Figure 3).

Hailey-Hailey disease is an autosomal dominant disorder that manifests in the third or fourth decades of life with an estimated prevalence of 1 in $50000 . .^{1}$ This genetic defect has been characterized on chromosome $3 \mathrm{q} 21$ as mutation in the ATP2Cl gene, which codes for an ATPdriven transmembrane calcium pump. ${ }^{2}$

About $15 \%$ of patients with Hailey-Hailey disease do not have a family history of the disease, possibly because of a sporadic gene mutation or because other family members have more mild, undiagnosed forms. ${ }^{3}$ Flexural and intertriginous areas are usually affected, and solitary involvement of the vulva is uncommon. Vesicles, papules, crusted erosions and plaques with a peripheral scaling border are typically present. When the disease is active, the plaques are velvety, hypertrophic and fissured, and they are often malodorous because of bacterial colonization. When

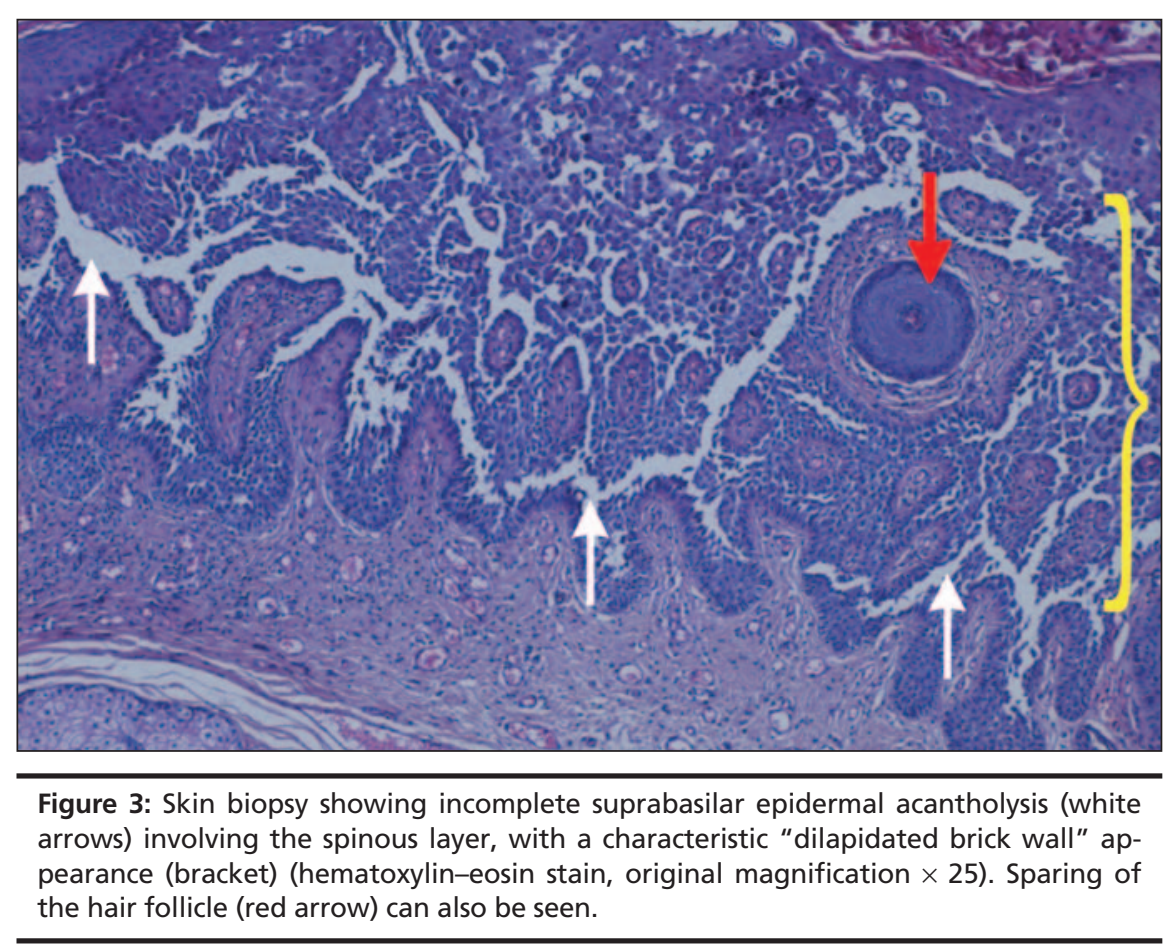

Figure 3: Skin biopsy showing incomplete suprabasilar epidermal acantholysis (white arrows) involving the spinous layer, with a characteristic "dilapidated brick wall" appearance (bracket) (hematoxylin-eosin stain, original magnification $\times 25$ ). Sparing of the hair follicle (red arrow) can also be seen.

the disease is less florid, dry scaly eczematous patches or crusted erosions predominate. Friction, heat, sweating and viral, fungal or bacterial infections exacerbate the condition.

The severity of Hailey-Hailey disease fluctuates over time and improves with age, and long remissions are possible. Asymptomatic longitudinal white bands affecting the nails are present in more than $70 \%$ of cases. ${ }^{1}$ This disease is frequently misdiagnosed as eczema or bacterial or fungal infections, because of temporary responses to topical corticosteroids combined with antibiotics or antifungal agents.

In our patient, the absence of Tzanck cells (multinucleated giant cells found in herpes simplex, varicella and pemphigus vulgaris) and the combination of history and fingernail bands argue against a diagnosis of herpes simplex. For a diagnosis of inverse psoriasis, one would have expected to see typical silvery scales of the hairy skin, which were not present.

In the early stages of Hailey-Hailey disease, the lesions can be misdiagnosed as erythrasma, a superficial infection with corynebacteria, identified by a coral-red fluorescence under a Wood lamp. Advanced lesions of Hailey-Hailey disease can also mimic pemphigo vulgaris; however, in pemphigo vulgaris, flaccid bullae have systemic and often mucous mani- festations. Other possible diagnoses that would be ruled out by histologic examination include extramammary Paget disease and erosive lichen planus.

There is a lack of agreement about the treatment of skin lesions caused by Hailey-Hailey disease. A range of therapeutic options have been described, including topical or systemic steroids with antibacterial agents, cyclosporine, retinoids, dapsone, methotrexate, thalidomide, PUVA (psoralen and UVA) or tacrolimus, tacalcitol and calcitriol. Preliminary reports have described favourable outcomes following treatment with photodynamic therapy with topical 5-aminolevulinic acid and subsequent irradiation with red light, dermabrasion, carbon dioxide laser ablation, electrodessication and cryosurgery. ${ }^{46}$ Bacterial colonization of active disease can be treated with antibiotics.

\section{Maria Le Donne MD}

Department of Gynecological,

Obstetric Sciences and Reproductive Medicine

University of Messina

Maria Lentini MD

Department of Human Pathology

University of Messina

Giovanna Moretti MD

Dermathology Unit

Papardo Hospital

\section{Serafinella Patrizia Cannavò MD}

Social and Environment Medicine

Dermatology Unit

University of Messina

Messina, Italy

This article has been peer reviewed.

Competing interests: None declared.

\section{REFERENCES}

1. Burge SM. Hailey-Hailey disease: the clinical features response to treatment and prognosis. $\mathrm{Br} J$ Dermatol 1992;126:275-82.

2. Hu Z, Bonifas JM, Beech J. Mutations in ATP2C1, encoding a calcium pump, cause Hailey-Hailey disease. Nat Genet 2000;24:61-5.

3. Tovell HM, Young AW. Disease of vulva: classification and incidence of 877 patients seen consecutively in vulva clinic. N Y State J Med 1977;77:938-41.

4. Umar SA, Bhattacharjee P, Brodell RT. Treatment of Hailey-Hailey disease with tacrolimus ointment and clobetasol propionate foam. J Drugs Dermatol 2004;3:200-3.

5. Ruiz-Rodriguez R, Alvarez JG, Jaèn P, et al. Photodynamic therapy with 5-aminolevulinic acid for recalcitrant familial benign pemphigus (Hailey-Hailey disease). J Am Acad Dermatol 2002;47:740-2.

6. Collet Villette AM, Richard MA, Fourquet F, et al. Treatment of Hailey-Hailey disease with carbon dioxide laser vaporization. Ann Dermatol Venereol 2005;132:637-40. 